spin speed considerably. The presence of liquid water on their surfaces would have helped but is not essential, viscous friction by bodily tides being sufficient.

As all things spin one would expect comets to spin too. This of course presupposes that comets have a large majority of their mass concentrated in a dirty snowball nucleus and not in a vast dust swarm of tiny particles (see News and Views 270, 558; 1977). Spining nuclei have been used to explain the non-gravitational motion of comets. Comet Encke has a period which is decreasing by 2.5 hours per orbital revolution. Comet Halley on the other hand persists in arriving late at perihelion by an average 4.1 days over the past 11 apparitions. Solar radiation causes the ice in the nucleus to evaporate. Molecules are ejected on the sunward side, generating a jet reaction. If the comet rotates the jet is displaced from the radial direction exerting a force on the nucleus which speeds it up if the spin is in the opposite direction to the orbital motion of the comet around the Sun. If the comet is spinning in the same sense as the planets the jet force slows down the comet, increasing its period and the semimajor axis of its orbit. As comets seem to have spin axes which are randomly orientated in space half seem to be accelerated and half decelerated. Unfortunately the acceleration or deceleration depends on the spin axis direction, spin period and the gas ejection rate. So measurement of this acceleration does not yield an unequivocal value for the spin rate.

Fred Whipple of the HarvardSmithsonian Center for Astrophysics, Cambridge, Massachusetts has been able to measure this spin rate using another technique. This is reported on page 134 of this issue of Nature. Whipple considers observations of Donati's comet which was discovered on 2 June 1858 by Giovanni B. Donati, a famous Italian astronomer and pioneer spectroscopist and observed for about 10 months. It has the reputation of being one of the most beautiful comets seen and had a most pronounced curved main tail. The period was thought to be around 2,000 years and it has been suggested that Donati is identical to the great comet recorded by the Roman writer Seneca in $146 \mathrm{BC}$.

One of the more unusual characteristics of Comet Donati was that its coma contained a series of haloes. These have been interpreted as expanding shells of material which seemed to be emitted with considerable violence from the central nuclear region. Haloes have also been seen in Comets Coggia (1974 III), Morehouse (1908 III) and Schwassmann-Wachmann I (1925 II). In Donati the haloes

\title{
Methanogenic bacteria
}

DR J. A. Steitz finds it difficult to accept ${ }^{1}$ our claim that methanogenic bacteria are representative of a group of organisms, the archaebacteria, that in a genealogical sense bear no more relationship to the typical bacteria than they do to (the "cytoplasmic aspect" of) the eukaryotes ${ }^{2-4}$. Citing a functionally defined pentanucleotide segment occurring very near the 3 . terminus of all bacterial $16 \mathrm{~S} \mathrm{rRNAs}^{5-8}$, but not found in eukaryotic $18 \mathrm{~S}$ rRNAs ${ }^{9-11}$, she concludes that "a high degree of functional relatedness between methanogenic and other $16 \mathrm{~S}$ rRNAs" exists", implying thereby that archaebacteria are specifically related to all other bacteria, and do not, therefore, represent a separate line of descent, distinct from the typical bacterial and the 'eukaryotic' lines ${ }^{3,4}$

What Steitz has done is to base a genealogical conclusion upon a single (simple) character - a procedure eschewed by systematists. Given three major lines of descent from a common ancestor, one would indeed expect to find numerous examples in which a single character is found in only two of the three lines. For this reason, classification should be, and is, based on multiple characters and/or composite characters such as cell wall ${ }^{12-13}$ or ribosomal $\mathrm{RNA}^{14}$ (in its entirety).

It is perhaps more convincing to give some specific examples: As pointed out originally, the usual tRNA common arm sequence, GT $\psi \mathrm{CG}$, is not found in archaebacteria $^{3}$, which might then suggest a "functional relatedness" between eukaryotic and typical bacterial tRNAs. Yet the initiator tRNA in (at least one of the) archaebacteria has characteristics that group it specifically with its eukaryotic counterparts $^{15}$. In terms of lipids, typical bacteria resemble eukaryotes rather than archaebacteria ${ }^{16-18}$. In terms of rRNA sizes and Steitz's criterion, typical bacteria and archaebacteria group together ${ }^{3,19}$. (A synopsis of archaebacteria and their characteristics will appear shortly ${ }^{20}$.)

Overall it seems that the number of molecular characteristics the archaebacteria share specifically with

were regular and sharp and Whipple assumes that the source of each halo is a massive mass ejection from a single active region on the cometary nucleus. This occurs every time that region becomes exposed to solar radiation, ejections being separated in typical bacteria is no greater than the number they share with eukaryotic cells. Moreover, archaebacteria possess striking features not found in either typical bacteria or eukaryotes.

We see no reason at present to rescind the conclusion that archaebacteria and typical bacteria represent separate lines of descent not related to one another specifically. Quite the opposite. All evidence accumulated pursuant to our initial discovery has tended to confirm and strengthen the case for the genealogical uniqueness of archaebacteria (see ref. 20 now in press).

\section{R. WOEse \\ Department of Genetics and Development, \\ University of Illinois at \\ Urbana-Champaign, \\ Illinois 61801}

\section{G. E. Fox}

Department of Biophysical Sciences, University of Houston,

Houston,

Texas 77004

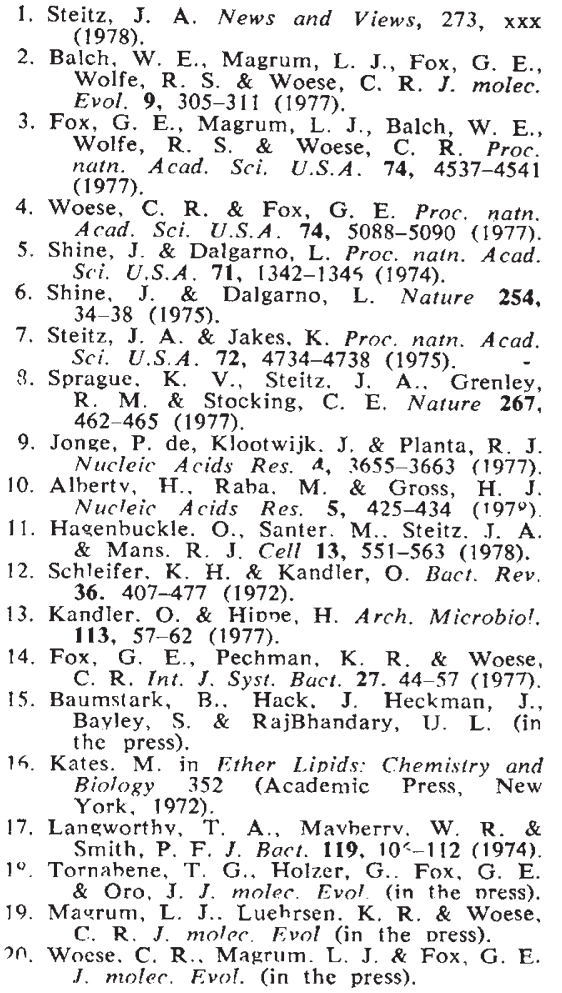

time by the cometary rotation period.

The ejected mass is moving away from the nucleus at a velocity which only depends on the distance between the comet and the Sun. Knowing the Earth-comet distance Whipple then calculates the spatial separation 